\title{
LITERACIA DIGITAL EM SAÚDE NA POPULACÃO PORTUGUESA, EM CONTEXTO PANDÉMICO: UM ESTUDO EMPÍRICO
}

\author{
Abílio Silva \\ Instituto Politécnico do Porto \\ Escola Superior de Saúde do Porto \\ abiliojsilva@gmail.com \\ Ana Isabel Martins \\ Instituto Politécnico do Porto \\ Escola Superior de Saúde do Porto \\ ana_ifmartins@hotmail.com \\ $\mathbf{M}^{\mathrm{a}}$ Helena Ferreira \\ Instituto Politécnico do Porto \\ Escola Superior de Saúde do Porto \\ mhelenap.ferreira@gmail.com \\ $M^{2}$ Isabel Ferreira \\ Instituto Politécnico do Porto \\ Escola Superior de Saúde do Porto \\ m.isabelpferreira@gmail.com \\ Mariana Vieira \\ Instituto Politécnico do Porto \\ Escola Superior de Saúde do Porto \\ marianavieira97@hotmail.com \\ Sara Silva Maia \\ Instituto Politécnico do Porto \\ Escola Superior de Saúde do Porto \\ saramaiafisio@gmail.com
}

Recepción Artículo: 17 mayo 2021 Admisión Evaluación: 17 mayo 2021 Informe Evaluador 1: 22 mayo 2021

Informe Evaluador 2: 23 mayo 2021 Aprobación Publicación: 02 junio 2021

\section{RESUMO}

Este estudo analisa o nível de literacia digital em saúde na população adulta portuguesa. Foi aplicado o questionário Digital Health Literacy (DHL) a uma amostra de 306 indivíduos. 0 instrumento está adaptado ao contexto pandémico atual e as dimensões estudadas são: pesquisa de informação, inclusão de conteúdo próprio, avaliação da fiabilidade e determinação da relevância informativa. 0 nível de literacia foi considerado suficiente e concluise que esta é influenciada pelas habilitações académicas ( $\mathrm{p}=0,013)$. Situação antagónica aconteceu com a idade ( $p=0326)$, o sexo ( $p=0,102)$, estatuto social $(p=0,230)$ e formação na área da saúde ( $p=0,147)$, cujas relações não apresentaram resultados estatisticamente significativos.

Palavras-chave: literacia em saúde; pandemia; literacia digital; Portugal; Covid-19 


\section{LITERACIA DIGITAL EM SAÚDE NA POPULAÇÃO PORTUGUESA, EM CONTEXTO PANDÉMICO: UM ESTUDO EMPIRICO}

\section{ABSTRACT}

Digital health literacy in portuguese population, in a pandemic context: an empirical study. We aim to evaluate the level of digital health literacy in portuguese adult population. It was applied a the Digital Health Literacy (DHL) survey to 306 individuals. The questionnaire assessed the following dimensions: information searching, adding self-generated content, evaluating reliability, and determining relevance. The level of literacy was considered sufficient and it is concluded that it is influenced by academic qualifications $(p=0.013)$. An antagonistic situation happened with age $(p=0326)$, sex $(p=0.102)$, social status $(p=0.230)$ and health education ( $p=0.147)$, whose relationships did not show statistically significant results.

Keywords: health literacy; pandemic; digital literacy; Portugal;Covid-19

\section{INTRODUÇÃO}

A Organização Mundial de Saúde (OMS) define literacia em saúde como o conjunto de "competências cognitivas e sociais e a capacidade da pessoa aceder, compreender e utilizar informação por forma a promover e a manter uma boa saúde" (Direção de Serviços de Prevenção da Doença e Promoção da Saúde, 2020)

A seleção da informação disponível sobre saúde, baseada no seu conhecimento, avaliação crítica e compreensão, capacita a população para a sua utilização e promove a sua autonomia no controlo das suas decisões quotidianas sobre cuidados de saúde. Vários autores referem a importância da literacia em saúde na prevenção da doença e promoção da saúde, mantendo ou melhorando a qualidade durante o ciclo de vida (Arriaga, M, Santos B, Silva A, Mata F, Chaves N, 2019; Espanha et al., 2016; Rosário et al., 2020). Uma inadequada literacia em saúde mostrou ser um indicador de: acesso inapropriado a cuidados de saúde, maior recurso a estes serviços, aumento dos custos de cuidados de saúde, aumento das taxas de mortalidade e maiores taxas de hospitalização (Haun et al., 2015; Palumbo et al., 2016).

0 aparecimento do novo coronavírus (SARS-CoV-2) veio trazer instabilidade e incerteza mundial. A importância da literacia digital em saúde merece especial destaque, tendo em conta que a informação disponível sobre esta pandemia é abundante, está em constante mudança e é acessível através de um largo espectro de tecnologias de informação digitais (Rosário et al., 2020). 0 rápido desenvolvimento da COVID-19, e a crise de saúde pública causada, provocou uma necessidade de repensar, rápida e criativamente, os modos de prestação de serviços de saúde e ir além dos modelos tradicionais, encorajando os recursos eletrónicos em saúde (Brørs et al., 2020; Crawford \& Serhal, 2020).0 desafio está nos entraves que ainda se encontram na população, como a falta de contacto com as novas tecnologias, falta de familiarização com os termos científicos e dificuldades em seguir as instruções de autocuidado (Brørs et al., 2020).

A OMS nomeia esta abundância de informação, como uma "infodemia", uma epidemia da informação, que tem vindo a acompanhar a pandemia COVID-19 (Dadaczynski et al., 2021; Kor et al., 2021; Zarocostas, 2020). A atual utilização massiva da internet abriu novas oportunidades para os indivíduos acederem a esta informação, ou desinformação, imediata relacionada com a saúde (Chong et al., 2020). A procura de esclarecimentos em relação à saúde, sem suporte, pode provocar alguma desorientação e consequente dispersão face à quantidade de informação obtida, levando a conclusões erradas ou irrelevantes (Brørs et al., 2020).

Numa sociedade que atravessa uma nova pandemia, o papel de cada indivíduo deve ser objeto de reflexão, advindo assim, o interesse para a concretização deste estudo. É esperado que indivíduos com mais literacia sobre o tema possam ter um maior envolvimento nas decisões em saúde e que, assim, se promovam melhores resultados em contexto pandémico (Rosário et al., 2020).

Urge a necessidade de potenciar conhecimento na literacia em saúde como ferramenta de saúde pública, ativando competências para assegurar que a informação disponível é, para além de corretamente filtrada, aplicada de forma a maximizar os ganhos e resultados em saúde, iniciando-se pela prevenção da doença e promoção do bem-estar. A Direção de Serviços de Prevenção da Doença e Promoção da Saúde (2020) refere no seu Plano de Ação para a Covid-19 que promover a literacia em saúde é decisivo para a implementação de medidas preventivas e para a própria evolução da pandemia. 
Com este estudo pretende-se avaliar o nível da literacia em saúde da população portuguesa em contexto pandémico, assim como entender se existe relação entre o nível de literacia e 0 sexo, idade, habilitações literárias, estatuto social, e formação na área da saúde.

\section{METODOLOGIA}

\section{Instrumento}

Foi aplicado o questionário DHL traduzido e validado para língua portuguesa em 2020 por Rosário et al., segundo as guidelines recomendadas pela OMS, e baseado em Van Der Vaart e Drossaert (2017) e adaptado ao contexto pandémico Covid-19. A devida autorização feita pela referida autora precedeu a utilização do questionário.

Neste questionário, com 15 itens, são avaliadas cinco dimensões relacionadas com a literacia digital em saúde no Covid-19: pesquisa de informação, inclusão de conteúdo próprio, avaliação da fiabilidade, determinação da relevância e proteção de privacidade (Rosário et al., 2020). 0 questionário está organizado segundo uma escala de Likert de 4 pontos: os inquiridos avaliam cada item numa escala de 1 a 4, em que "1 - muito difícil" e "4 - muito fácil". Quanto mais alta a pontuação obtida numa pergunta, mais alto o nível de literacia. A escala possui uma boa validade de construto e conteúdo (Van Der Vaart \& Drossaert, 2017).

0 valor médio teórico foi calculado para a pontuação total, assim como, para cada uma das dimensões. Para posterior análise a variável da pontuação total foi dividida em literacia suficiente ou limitada, seguindo o valor da média teórica.

Para além dos itens constantes no questionário DHL, foram também aplicadas questões sociodemográficas, por serem consideradas de relevo. Foi utilizada a escala de MacArthur para aferir a perceção onde o inquirido se enquadra relativamente ao seu grau de literacia em saúde no Covid-19 e ao seu estatuto social, que retrata uma escada de 10 degraus (Adler et al., 2000). Os participantes foram incitados a responder qual o degrau que melhor reflete 0 seu conhecimento sobre a pandemia e sobre o seu estatuto numa sociedade hierárquica, em que valores mais altos correspondem a maior literacia ou estatutos sociais mais elevados, respetivamente.

0 questionário foi aplicado a maiores de 18 anos e com nacionalidade portuguesa, através da plataforma GoogleForms e difundido nas redes sociais pela técnica de amostragem bola de neve. 0 período de disponibilização online decorreu entre os dias 25 de março de 2021 (com 819210 casos de COVID-19 positivos confirmados pela DGS) e 12 de abril (com 827765 casos confirmados pela DGS). Obteve-se o consentimento informado para divulgação dos dados, com garantia de confidencialidade e anonimização.

Relativamente à fiabilidade, 0 de Cronbaché de $0,890,0$ que indica uma boa consistência interna. Esta consistência foi conseguida após a eliminação dos itens 13, 14 e 15 (dimensão proteção da privacidade), tal como no estudo realizado por Rosário et al. (2020), a última dimensão foi excluída da análise. 0 de Cronbach para as dimensões foi de 0,768 (pesquisa de informação), 0,877 (inclusão de conteúdo próprio), 0,750 (avaliação da fiabilidade) e 0,792 (determinação da relevância). Todos os valores são aceitáveis e acima do recomendado (Marôco, 2018).

\section{Participantes}

0 inquérito foi aplicado a um total de 306 participantes cuja caracterização sociodemográfica se encontra representada na tabela 1. 


\section{LITERACIA DIGITAL EM SAÚDE NA POPULAÇÃO PORTUGUESA, EM CONTEXTO PANDÉMICO:}

UM ESTUDO EMPIRICO

Tabela 1. Características sociodemográficas dos participantes do estudo ( $N=306)$.

\begin{tabular}{|c|c|c|}
\hline Variáveis & $\mathbf{n}$ & $\%$ \\
\hline \multicolumn{3}{|l|}{ Sexo } \\
\hline Feminino & 82 & 26,8 \\
\hline Masculino & 224 & 73,2 \\
\hline \multicolumn{3}{|l|}{ Idade } \\
\hline $20-29$ anos & 87 & 28,4 \\
\hline 30-39 anos & 94 & 30,7 \\
\hline 40-49 anos & 71 & 23,2 \\
\hline$\geq 50$ anos & 51 & 17,6 \\
\hline Média ( \pm desvio-padrão) & \multicolumn{2}{|c|}{$37,97( \pm 11,604)$ anos } \\
\hline \multicolumn{3}{|l|}{ Habilitações Académicas } \\
\hline $\begin{array}{c}\text { Até ensino secundário ou } \\
\text { equivalente }\end{array}$ & 77 & 25,2 \\
\hline Ensino Superior & 229 & 74,8 \\
\hline \multicolumn{3}{|l|}{ Estatuto Social } \\
\hline Baixo & 30 & 9,8 \\
\hline Médio & 216 & 70,6 \\
\hline Alto & 60 & 19,6 \\
\hline Mediana & \multicolumn{2}{|c|}{6} \\
\hline
\end{tabular}

A média \pm desvio padrão do score total de literacia em saúde é de 37,77×5,167 (com um mínimo teórico de 15 e um máximo teórico de 48). Dos 306 participantes que aceitaram responder ao questionário, 94 indivíduos foram excluídos do tratamento estatístico por não terem efetuado pesquisa sobre o novo coronavírus nas quatro semanas que antecederam a resposta ao questionário. Portanto, foram incluídos um total de 212 indivíduos na análise estatística.

0 estatuto social foi dividido em estatuto social baixo (1 a 4), médio (5 a 7 ) e elevado (8 a 10). Para analisarmos a literacia em saúde relacionada com a idade, procedemos à separação das idades em quatro classes, sendo elas dos 20 aos 29 ( $n=87)$, dos 30 aos 39 anos ( $n=94)$, dos 40 aos 49 ( $n=71)$ e igual ou superior a 50 anos $(n=54)$.

As habilitações académicas foram dividas em duas classes: até ao ensino secundário ou equivalente ( $n=41)$ e ensino superior $(n=171)$.

\section{Análise estatística}

0 tratamento dos dados foi feito com recurso ao software estatístico IBM SPSS versão 27.0. A análise estatística descritiva foi feita para caracterizar a amostra, incluindo dados sociodemográficos e comportamentais. $\mathrm{Na}$ análise inferencial recorreu-se a testes de hipóteses: teste t para uma amostra; teste t para amostras independentes. Para variáveis que não seguem a normalidade usou-se Kruskal-Wallis. Por último recorreu-se ainda ao teste de Fisher. 0 nível de significância considerado foi de 0,05.

\section{RESULTADOS E DISCUSSÃO}

\section{Caracterização da literacia em saúde na população portuguesa e suas dimensões}

Com 0 objetivo de estudar a literacia aplicou-se 0 teste t para uma amostra, através da pontuação total. É possível assumir a normalidade da distribuição amostral da média da variável (teorema do limite central), uma vez que $n$ superior a 30 ( $n=212$ ). Foi também realizado o teste t para uma amostra, por forma a definir se a literacia 
é suficiente (com valores superiores a 30) ou limitada (com valores inferiores ou iguais a 30) da população portuguesa. Após análise, deduziu-se que a média da pontuação total é significativamente superior a 30, ( $p<0,001)$. A população estudada demonstrou ter uma literacia digital em saúde suficiente. No entanto, este resultado não vai ao encontro de outros estudos realizados na área em Portugal (Arriaga, M, Santos B, Silva A, Mta F, Chaves N, 2019; Pedro et al., 2016).

Como se observa na tabela 2, avaliando os itens com uma escala de Likert com 4 pontos, a população portuguesa apresenta um valor de pontuação total superior ao valor da média teórica, o que expressa um nível de literacia suficiente. Relativamente às dimensões, todas elas se encontram acima do valor da média teórica' $(7,5)$.

Tabela 2. Estatística descritiva para as dimensões e a pontuação total.

\begin{tabular}{ccc}
\cline { 2 - 3 } & Média & Desvio-padrão \\
\hline Pesquisa de informação & 9,56 & 1,421 \\
\hline Inclusão de conteúdo próprio & 9,02 & 2,043 \\
\hline Avaliação de fiabilidade & 9,42 & 1,563 \\
\hline Determinação da relevância & 9,77 & 1,434 \\
\hline Pontuação Total & 37,77 & 5,167 \\
\hline
\end{tabular}

\section{Determinantes da literacia}

\section{Idade}

Para analisar a literacia relacionada com a idade, procedeu-se à separação das idades em quatro classes como descrito em epígrafe. Através do teste de Kolmogorov-Smirnov, não se verificou o pressuposto da normalidade da variável em estudo para as classes [20;29] com $p=0,002$, [30;39] com $p<0,0001$ e para idades iguais ou superiores a 50 anos com $\mathrm{p}=0,020$.

Utilizou-se 0 teste Kruskal-Wallis para esta análise. Não se encontram evidências que comprovem que a literacia varia em função da idade $(\mathrm{p}=0,266)$.

Outros estudos não corroboram os nossos resultados, sugerindo que existe relação entre a literacia e a idade. Ainda assim, os resultados não demonstram ser consensuais, havendo estudos que demonstram que a literacia diminuiu com a idade (Berens et al., 2016; Espanha et al., 2016; Guo et al., 2021); embora outros estudos não consigam evidenciar esta relação (Dadaczynski et al., 2021).

\section{Sexo}

Com o intuito de verificar se a literacia é influenciada pelo sexo, aplicou-se o teste t para duas amostras independentes, tanto para pontuação total, como para as diferentes componentes. 0 teste Fisher foi utilizado para verificar a existência de associação entre um nível de literacia suficiente ou limitada com o sexo.

É verificado o pressuposto da normalidade da distribuição da média da variável (teorema do limite central), uma vez que $n$ é superior a 30 em ambos os grupos ( $n_{\text {maculino }}=60$ e $n_{\text {feminino }}=152$ ).

Uma vez que a dimensão das amostras é diferente nos dois grupos, aplicou-se 0 teste de Levene para as igualdades das variâncias na pontuação total $(p=0,790)$, na dimensão de pesquisa de informação $(p=0,167)$, inclusão de conteúdo próprio $(p=0,437)$, avaliação da fiabilidade $(p=0,179)$ e determinação da relevância $(\mathrm{p}=0,134)$.

Tanto para a pontuação total $(p=0,102)$, como para as dimensões: pesquisa de informação $(p=0,475)$, inclusão de conteúdo próprio $(p=0,687)$ e determinação da relevância $(p=0,054)$, não existem evidências que a literacia em saúde seja diferente entre homens e mulheres. № entanto, na dimensão da avaliação da fiabilidade existem evidências estatisticamente significativas que comprovam diferenças na literacia consoante e sexo $(p=0,014)$. 


\section{LITERACIA DIGITAL EM SAÚDE NA POPULAÇÃO PORTUGUESA, EM CONTEXTO PANDÉMICO: UM ESTUDO EMPIRICO}

A utilização do teste de Fisher é fundamentada pela não verificação dos pressupostos de aplicabilidade do Qui-quadrado. Os dados não evidenciam a existência de associação entre a literacia suficiente ou limitada relativamente ao sexo $(\mathrm{p}=0,072)$.

Os resultados obtidos são equiparáveis ao do estudo realizado por Berens et al. (2016) em que não se verificou diferença estatística significativa entre o sexo masculino e feminino. Outros autores como Dadaczynski et al. (2021) encontram relação, onde num estudo com estudantes alemães, o sexo feminino revelou ter menores níveis de literacia digital em saúde. Um outro estudo feito por Rosário et al. (2020) em estudantes universitários portugueses mostrou níveis de literacia em saúde significativamente mais altos nos alunos do sexo masculino. Por outro lado, um estudo feito na austrália por Dodd et al. (2021), demonstrou que estudantes do sexo feminino têm literacia mais alta em relação a alunos do sexo masculino.

\section{Estatuto Social}

Para analisar a influência do estatuto social na literacia em saúde, utilizamos o teste Kruskal-Wallis, uma vez que não se verificou o pressuposto da normalidade da variável em estudo, através de Kolmogorov-Smirnov, para as classes média $(p<0,001)$ e alta $(\mathrm{p}=0,039)$.

Os resultados demonstram que não existem evidências estatísticas para afirmar que a literacia em saúde da população portuguesa não é influenciada pelo estatuto social $(\mathrm{p}=0,230)$. Após análise dos resultados por regressão linear, com p=0,124, não se verifica correlação. Os resultados alcançados não corroboram outros estudos realizados na área, onde os níveis altos de literacia acompanham a posição socioeconómica da população estudada (Berens et al., 2016; Rosário et al., 2020).

\section{Habilitações Académicas}

0 recurso ao teste t para duas amostras independentes teve o propósito de relacionar as habilitações académicas com a literacia em saúde. Assumiu-se normalidade da distribuição amostral da média da variável (teorema do limite central), uma vez que $n$ superior a 30 em ambos os grupos ( $n_{\text {até ensino secundário }}=41$ e $n_{\text {ensino supe- }}$ rior $=171)$. Sendo que a dimensão das amostras é diferente nos dois grupos, aplicou-se 0 teste de Levene para as igualdades das variâncias na pontuação total $(p=0,130)$.

Existem evidências estatísticas suficientes para afirmar que a literacia em saúde é diferente entre indivíduos com diferentes graus habilitacionais $(\mathrm{p}=0,013)$. Outro estudo realizado em Portugal confirma que níveis elevados de escolaridade tendem a corresponder a níveis elevados de literacia em saúde (Espanha et al., 2016). Estes dados são também reforçados por estudos realizados internacionalmente (Guo et al., 2021; Neter \& Brainin, 2012; Spring, 2020).

\section{Formação na área da saúde}

Para avaliar se a literacia em saúde é influenciada pela formação prévia na área da saúde, foi utilizado o teste t para duas amostras independentes. Assumiu-se normalidade da distribuição amostral da média da variável (teorema do limite central), uma vez que n superior a 30 em ambos os grupos ( $n_{\text {com formação em saúde }}=123$ e $n_{\text {sem }}$ formação em saúde $=89$ ). Sendo que a dimensão das amostras é diferente nos dois grupos, aplicou-se o teste de Levene para as igualdades das variâncias na pontuação total $(p=0,249)$. Não encontramos relação entre a literacia em saúde e a formação na área da saúde $(p=0,147)$. Não foram encontrados estudos que comprovem esta relação.

\section{LIMITAÇÕES}

0 diminuto tempo de recolha dos dados, e consequentemente a pequena dimensão amostral pouco representativa da população portuguesa, pode não refletir verdadeiramente o grau de literacia. A divulgação do questionário, com recurso a técnica de amostragem por bola-de-neve, pode revelar ser uma limitação ao estudo por enviesar a amostra abrangida. 


\section{CONCLUSÕES}

A literacia em saúde permite 0 empoderamento da população, assim como a sua capacitação para a tomada de decisão informada sobre saúde. Geralmente, indivíduos com níveis superiores de literacia, conseguem fazer uma melhor gestão da sua condição de saúde.

Neste estudo verificou-se um nível de literacia em saúde digital suficiente da população portuguesa, e que apenas as habilitações académicas influenciam a literacia em contexto pandémico $(p=0,013)$. Foram ainda avaliados outros determinantes como a idade $(p=0326)$, 0 sexo $(p=0,102)$, estatuto social $(p=0,230)$ e formação na área da saúde $(\mathrm{p}=0,147)$, onde se concluiu não existir relação estatisticamente significativa com nenhum.

Aconselha-se a realização de mais estudos na área, devido aos resultados discordantes, existentes na literatura encontrada.

\section{REFERÊNCIAS BIBLIOGRÁFICAS}

Adler, N. E., Epel, E. S., Castellazzo, G., \& Ickovics, J. R. (2000). Relationship of subjective and objective social status with psychological and physiological functioning: Preliminary data in healthy white women. Health Psychology, 19(6), 586-592. https://doi.org/10.1037/0278-6133.19.6.586

Arriaga, M, Santos B, Silva A, Mata F, Chaves N, F. G. (2019). Plano de ação para a literacia em saúde. Direção Geral Da Saúde, 6. https://www.dgs.pt/em-destaque/programa-nacional-de-educacao-para-a-saude-literacia-e-autocuidados.aspx

Berens, E. M., Vogt, D., Messer, M., Hurrelmann, K., \& Schaeffer, D. (2016). Health literacy among different age groups in Germany: results of a cross-sectional survey. BMC Public Health, 16(1), 1-8. https://doi.org/10.1186/s12889-016-3810-6

Brørs, G., Norman, C. D., \& Norekvål, T. M. (2020). Accelerated importance of eHealth literacy in the COVID-19 outbreak and beyond. In European Journal of Cardiovascular Nursing (Vol. 19, Issue 6, pp. 458-461). SAGE Publications Inc. https://doi.org/10.1177/1474515120941307

Chong, Y. Y., Cheng, H. Y., Chan, H. Y. L., Chien, W. T., \& Wong, S. Y. S. (2020). COVID-19 pandemic, infodemic and the role of eHealth literacy. In International Journal of Nursing Studies (Vol. 108, p. 103644). Elsevier Ltd. https://doi.org/10.1016/j.ijnurstu.2020.103644

Crawford, A., \& Serhal, E. (2020). Digital Health Equity and COVID-19: The Innovation Curve Cannot Reinforce the Social Gradient of Health. J Med Internet Res, 22(6), 19361. https://doi.org/10.2196/19361

Dadaczynski, K., Okan, O., Messer, M., Leung, A. Y. M., Rosário, R., Darlington, E., \& Rathmann, K. (2021). Digital Health Literacy and Web-Based Information-Seeking Behaviors of University Students in Germany During the COVID-19 Pandemic: Cross-sectional Survey Study. Journal of Medical Internet Research, 23(1), e24097. https://doi.org/10.2196/24097

Direção de Serviços de Prevenção da Doença e Promoção da Saúde. (2020). Literacia Em Saúde E a Covid-19: Plano, Prática e Desafios. 18.

Dodd, R. H., Dadaczynski, K., Okan, 0., McCaffery, K. J., \& Pickles, K. (2021). Psychological wellbeing and academic experience of university students in australia during covid-19. International Journal of Environmental Research and Public Health, 18(3), 1-12. https://doi.org/10.3390/ijerph18030866

Espanha, R., Ávila, P., \& Mendes, R. V. (2016). Literacia em saúde em Portugal- relatório síntese. Fundação Calouste Gulbenkian, 1-16. http://www.gulbenkian.pt

Guo, Z., Zhao, S. Z., Guo, N., Wu, Y., Weng, X., Wong, J. Y.-H., Lam, T. H., \& Wang, M. P. (2021). Socioeconomic Disparities in eHealth Literacy and Preventive Behaviors During the COVID-19 Pandemic in Hong Kong: Cross-sectional Study. Journal of Medical Internet Research, 23(4), e24577. https://doi.org/10.2196/24577

Haun, J. N., Patel, N. R., French, D. D., Campbell, R. R., Bradham, D. D., \& Lapcevic, W. A. (2015). Association between health literacy and medical care costs in an integrated healthcare system: A regional population based study. BMC Health Services Research, 15(1), 1-11. https://doi.org/10.1186/s12913-015-0887-z 
Kor, P. P. K., Leung, A. Y. M., Parial, L. L., Wong, E. M. L., Dadaczynski, K., Okan, O., Amoah, P. A., Wang, S. S., Deng, R., Cheung, T. C. C., \& Molassiotis, A. (2021). Are People With Chronic Diseases Satisfied With the Online Health Information Related to COVID-19 During the Pandemic? Journal of Nursing Scholarship, 53(1), 75-86. https://doi.org/10.1111/jnu.12616

Marôco, J. (2018). Análise Estatística com o SPSS Statistics.: $7^{a}$ edição. ReportNumber, Lda.

Neter, E., \& Brainin, E. (2012). eHealth literacy: Extending the digital divide to the realm of health information. Journal of Medical Internet Research, 14(1). https://doi.org/10.2196/jmir.1619

Palumbo, R., Annarumma, C., Adinolfi, P., Musella, M., \& Piscopo, G. (2016). The Italian Health Literacy Project: Insights from the assessment of health literacy skills in Italy. Health Policy, 120(9), 1087-1094. https://doi.org/10.1016/j.healthpol.2016.08.007

Pedro, A. R., Amaral, 0., \& Escoval, A. (2016). Literacia em saúde, dos dados à ação: tradução, validação e aplicação do European Health Literacy Survey em Portugal. Revista Portuguesa de Saude Publica, 34(3), 259275. https://doi.org/10.1016/j.rpsp.2016.07.002

Rosário, R., Martins, M. R. O., Augusto, C., Silva, M. J., Martins, S., Duarte, A., Fronteira, I., Ramos, N., Okan, 0., \& Dadaczynski, K. (2020). Associations between covid-19-related digital health literacy and online information-seeking behavior among portuguese university students. International Journal of Environmental Research and Public Health, 17(23), 1-11. https://doi.org/10.3390/ijerph17238987

Spring, H. (2020). Health literacy and COVID-19. Health Information and Libraries Journal, 37(3), 171-172. https://doi.org/10.1111/hir.12322

Van Der Vaart, R., \& Drossaert, C. (2017). Development of the digital health literacy instrument: Measuring a broad spectrum of health 1.0 and health 2.0 skills. Journal of Medical Internet Research, 19(1), 0-13. https://doi.org/10.2196/jmir.6709

Zarocostas, J. (2020). How to fight an infodemic. Lancet (London, England), 395(10225), 676. https://doi.org/10.1016/S0140-6736(20)30461-X

Tabela 1. Características sociodemográficas dos participantes do estudo ( $N=306)$.

\begin{tabular}{|c|c|c|}
\hline Variáveis & $\mathbf{n}$ & $\%$ \\
\hline \multicolumn{3}{|l|}{ Sexo } \\
\hline Feminino & 82 & 26,8 \\
\hline Masculino & 224 & 73,2 \\
\hline \multicolumn{3}{|l|}{ Idade } \\
\hline 20-29 anos & 87 & 28,4 \\
\hline 30-39 anos & 94 & 30,7 \\
\hline $40-49$ anos & 71 & 23,2 \\
\hline$\geq 50$ anos & 51 & 17,6 \\
\hline Média ( \pm desvio-padrão) & \multicolumn{2}{|c|}{$37,97( \pm 11,604)$ anos } \\
\hline \multicolumn{3}{|l|}{ Habilitações Académicas } \\
\hline $\begin{array}{c}\text { Até ensino secundário ou } \\
\text { equivalente }\end{array}$ & 77 & 25,2 \\
\hline Ensino Superior & 229 & 74,8 \\
\hline \multicolumn{3}{|l|}{ Estatuto Social } \\
\hline Baixo & 30 & 9,8 \\
\hline Médio & 216 & 70,6 \\
\hline Alto & 60 & 19,6 \\
\hline Mediana & \multicolumn{2}{|c|}{6} \\
\hline
\end{tabular}


SUPERACIÓN EN POSITIVO Y APRENDIZAJE: LA RESPUESTA AL COVID-19

Tabela 2. Estatística descritiva para as dimensões e a pontuação total.

\begin{tabular}{ccc}
\cline { 2 - 3 } & Média & Desvio-padrão \\
\hline Pesquisa de informação & 9,56 & 1,421 \\
\hline Inclusão de conteúdo próprio & 9,02 & 2,043 \\
\hline Avaliação de fiabilidade & 9,42 & 1,563 \\
\hline Determinação da relevância & 9,77 & 1,434 \\
\hline Pontuação Total & 37,77 & 5,167 \\
\hline
\end{tabular}

\section{NOTAS}

${ }^{1}$ Sendo que o mínimo teórico é 4 e o máximo teórico é 12 
